\title{
Recent LHC/TOTEM data challenging the standard Regge pole theory
}

\author{
Norbert Bence ${ }^{1, *}$, László Jenkovszky ${ }^{2, * *}$, and István Szanyi ${ }^{1, * * *}$ \\ ${ }^{1}$ Uzhgorod National University, 14, Universytets'ka str., Uzhgorod, 88000, Ukraine \\ ${ }^{2}$ Bogolyubov Institute for Theoretical Physics (BITP), Ukrainian National Academy of Sciences 14-b, \\ Metrologicheskaya str., Kiev, 03680, Ukraine
}

\begin{abstract}
We analyse the recently discovered phenomena in elastic protonproton scattering at the LHC: the low- $|t|$ "break" (departure from the exponential behaviour of the diffraction cone), the accelerating rise with energy of the forward slope $B(s)$, the absence of secondary dips and bumps on the cone and the role of the odderon in the forward phase of the amplitude, $\rho(13 \mathrm{TeV})=0.09 \pm 0.01$, especially its contribution at the dip region, measured recently by TOTEM. The dip at $13 \mathrm{TeV}$ seems to become more shallow with respect to lower energies, and we attribute this important new phenomena to the odderon contribution. These findings call for a revision of the standeard Regge-pole theory.
\end{abstract}

\section{Introduction}

During the past seven years the TOTEM Collaboration produced a number of spectacular results on proton-proton elastic and total cross sections measured at the LHC in the range $2.76 \leq \sqrt{s} \leq 13 \mathrm{TeV}$ [1]. While the total, $\sigma_{\text {tot }}$, integrated elastic, $\sigma_{e l}$ and inelastic, $\sigma_{\text {in }}$ cross sections, in general follow the expectations and extrapolations from lower energies, several new, unexpected features were discovered in elastic scattering. These are:

1. low- $|t|$ structure in $d \sigma / d t$ (the "break") [2, 3];

2. absence of secondary dips/bumps in $d \sigma / d t[3,4]$;

3. further dynamics (deepening or filling-in?) of the dip; first results at $13 \mathrm{TeV}$, reported by TOTEM;

4. unexpected rapid rise of the forward slope $B(s, 0))[1]$;

5. surprisingly low value of the phase of the forward amplitude [3].

We analyze these phenomena within a Regge pole model and show that they call for a revision of the standard model based on a simple Regge pole (pomeron) lying on a linear trajectory with subsequent eikonalization.

In the present paper we analyze elastic proton-proton scattering with special emphasis on the above issues, discovered by TOTEM, partly confirmed also by ATLAS [5, 6].

\footnotetext{
*e-mail: bencenorbert007@gmail.com

**e-mail: jenk@bitp.kiev.ua

***e-mail: sz.istvan03@gmail.com
} 


\section{The dipole model and fits to the data}

We present the dipole pomeron model (for a review see e.g. [7]), main tool in our analysis, supplemented by preliminary fits to high-energy forward $p p$ observables (elastic, inelastic and total cross sections). The DP pomeron model is the unique alternative to a simple pole, since higher order poles are not allowed by unitarity. Furthermore, the DP produces (logarithmically) rising cross sections even at unit intercept. The DP scales reproducing itself with respect to $s$-channel unitarity corrections. A particularly attractive feature of the DP is the built-in mechanism of the diffraction pattern: a single minimum appears, followed by a maximum in the differential cross section, confirmed by the experimental data in a wide span of energies.

The scattering amplitude is [8]:

$$
A(s, t)_{p p}^{\bar{p} p}=A_{P}(s, t)+A_{f}(s, t) \pm\left[A_{\omega}(s, t)+A_{O}(s, t)\right] .
$$

Secondary reggeons are parametrized in a standard way, with linear Regge trajectories and exponential residua.

As already mentioned, the pomeron is a dipole in the $j$-plane

$$
\begin{aligned}
& A_{P}(s, t)=\frac{d}{d \alpha_{P}}\left[\mathrm{e}^{-i \pi \alpha_{P} / 2} G\left(\alpha_{P}\right)\left(s / s_{0 P}\right)^{\alpha_{P}}\right]= \\
& \mathrm{e}^{-i \pi \alpha_{P}(t) / 2}\left(s / s_{0 P}\right)^{\alpha_{P}(t)}\left[G^{\prime}\left(\alpha_{P}\right)+\left(L_{P}-i \pi / 2\right) G\left(\alpha_{P}\right)\right] .
\end{aligned}
$$

Since the first term in squared brackets determines the shape of the cone, one fixes

$$
G^{\prime}\left(\alpha_{P}\right)=-a_{P} \mathrm{e}^{b_{P}\left[\alpha_{P}-1\right]},
$$

where $G\left(\alpha_{P}\right)$ is recovered by integration. Consequently the pomeron amplitude Eq.(2) may be rewritten in the following "geometrical" form (for details see [7] and references therein):

$$
A_{P}(s, t)=i \frac{a_{P} s}{b_{P} s_{0 P}}\left[r_{1 P}^{2}(s) \mathrm{e}^{r_{1 P}^{2}(s)\left[\alpha_{P}-1\right]}-\varepsilon_{P} r_{2 P}^{2}(s) \mathrm{e}^{r_{2 P}^{2}(s)\left[\alpha_{P}-1\right]}\right],
$$

where $r_{1 P}^{2}(s)=b_{P}+L_{P}-i \pi / 2, r_{2 P}^{2}(s)=L_{P}-i \pi / 2, L_{P} \equiv \ln \left(s / s_{0 P}\right)$ and the pomeron trajectory:

$$
\alpha_{P} \equiv \alpha_{P}(t)=1+\delta_{P}+\alpha_{P}^{\prime} t
$$

The odderon contribution is assumed to be of the same form as that of the pomeron apart from different values of adjustable parameters (labeled by the subscript " $O$ ") and the negative charge parity.

In earlier versions of the DP, to avoid conflict with the Froissart bound, the intercept of the pomeron was fixed at $\alpha(0)=1$. However later it was realized that the logarithmic rise of the total cross sections provided by the DP may not be sufficient to meet the data, therefore a supercritical intercept was allowed for. From the fits to the data the value $\delta=\alpha(0)-1 \approx 0.04$, half of Landshoff's value [9] follows. This is understandable: the DP promotes half of the rising dynamics, thus moderating the departure from unitarity at the "Born" level (smaller unitarity corrections).

We use the norm where

$$
\frac{d \sigma_{e l}}{d t}(s, t)=\frac{\pi}{s^{2}}|A(s, t)|^{2} \text { and } \sigma_{t o t}(s)=\frac{4 \pi}{s} \mathfrak{J} m A(s, t=0) .
$$


The parameter $\rho(s)$, the ratio of the real and imaginary part of the forward scattering amplitude is

$$
\rho(s)=\frac{\operatorname{ReA}(s, t=0)}{\operatorname{ImA}(s, t=0)}
$$

The free parameters of the model defined by the formulas Eqs. (1-7) were fitted simultaneously to the following dataset:

- TOTEM $7 \mathrm{TeV}$ elastic $p p$ differential cross section data [4] in the region of $0.35 \leqslant|t| \leqslant 2.5$ $\mathrm{GeV}^{2}$;

- SPS 546 and $630 \mathrm{GeV}$ elastic $p \bar{p}$ differential cross section data [10] in the region of $0.5 \leqslant$ $|t| \leqslant 2.2 \mathrm{GeV}^{2}$

- $p p$ and $p \bar{p}$ total cross section and $\rho$ parameter data $[1,3,4,11-15]$ in the region of $20 \leqslant$ $\sqrt{s} \leqslant 57000 \mathrm{GeV}$.

\subsection{Total cross section, $\rho$-parameter and elastic slope}

Fig. 1 shows the results of our fits to $p p$ and $p \bar{p}$ total cross section data $[1,11-13,15]$.

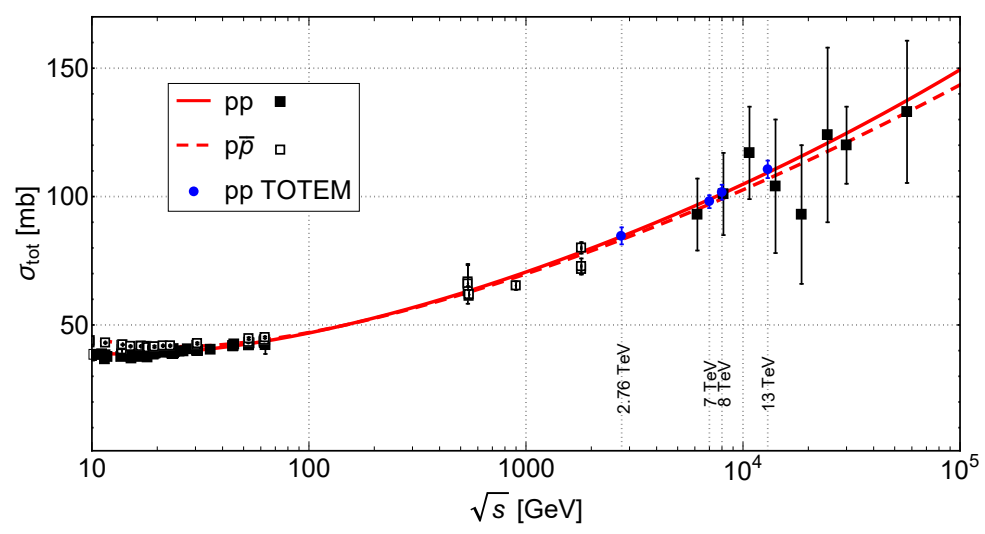

Figure 1. Fits to $p p$ and $p \bar{p}$ total cross section data $[1,11-13,15]$ using the model Eqs. (1-7).

The widely discussed recent measurement of the phase $\rho(13 \mathrm{TeV})=0.09 \pm 0.01$ (or $\rho(13 \mathrm{TeV})=0.1 \pm 0.01)$ [3]. This data point lies well below the expectations (extrapolations) from lower energies, although this should not be dramatized. The flexibility of the odderon parametrization leaves room for perfect fits to this data point simultaneously fitting the total cross section as (see below). More critical the inclusion of non-forward data, both for $p p$ and $\bar{p} p$ especially around the dip region, to which the odderon is sensitive! Independent of theoretical interpretation, another, independent measurement of the phase is highly desirable. 


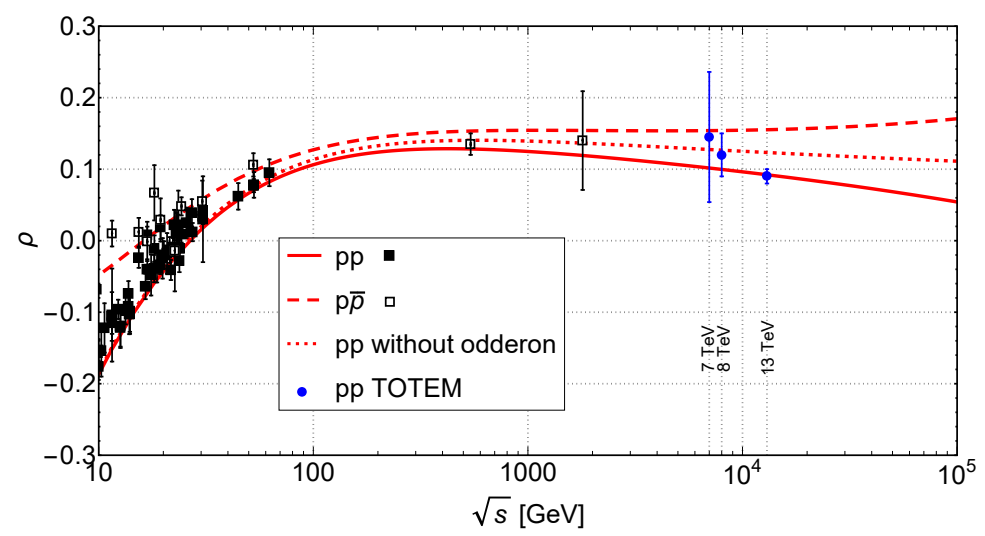

Figure 2. Fits to $p p$ and $p \bar{p}$ ratio $\rho$ data $[3,4,12,14]$ using the model Eqs. (1-5) and Eq. 7.

Fig. 2 shows the results of our fits to $p p$ and $p \bar{p}$ total cross section data $[3,4,12,14]$. Our model simultaneously can describe the new TOTEM measurement on the total cross section and the parameter- $\rho$, however only a few model does [3]. In Ref. [16] we managed to achieve similar good results by using simple pomeron and odderon poles. As we can see in Fig. 2 the case without the odderon (presented with the dotted line) does not give a description for the new $13 \mathrm{TeV}$ data point. However the neglection of the oddereon has no significant effect for the description of the new TOTEM total cross section measurements.

The above calculations are intended to provide the ground for our study of the slope, to be presented in the next Section.

The slope is defined as

$$
B(s, t=0)=\frac{d}{d t}\left(\left.\ln \frac{d \sigma}{d t}(s, t)\right|_{t=0} .\right.
$$

While in Regge-pole models the rise of the total cross sections is regulated by the hardness of the Regge pole (here, the pomeron), the slope $B(s)$ in case of a single and simple Regge pole is always logarithmic. Deviation (acceleration) may arise from more complicated Regge singularities, the odderon and/or from unitarity corrections.

With the model and its fitted parameters in hand, we calculated the $p p$ and $p \bar{p}$ elastic slope $B(s)$ using Eq. 8. Result is shown in Fig. 3. More detail from the slope given by Eq. (8) with the norm Eq. (6) and the amplitude Eq. (1) can be find in the Appendix.

By using the parametrization

$$
B(s)=b_{0}+b_{1} \ln \left(s / s_{0}\right)+b_{2} \ln ^{2}\left(s / s_{0}\right)
$$

we did a fit to the $p p$ and $p \bar{p}$ elastic slope data in the energy region of $546 \leqslant \sqrt{s} \leqslant 13000$ $\mathrm{GeV}$. Neglecting the lower, outlier $546 \mathrm{GeV}$ data points the fitted values of parameters $b_{0}=$ $13.75, b_{1}=-0.358, b_{2}=0.0379$ and $\chi^{2} / N D F=1.97$. Its result is also shown in Fig. 3 . 


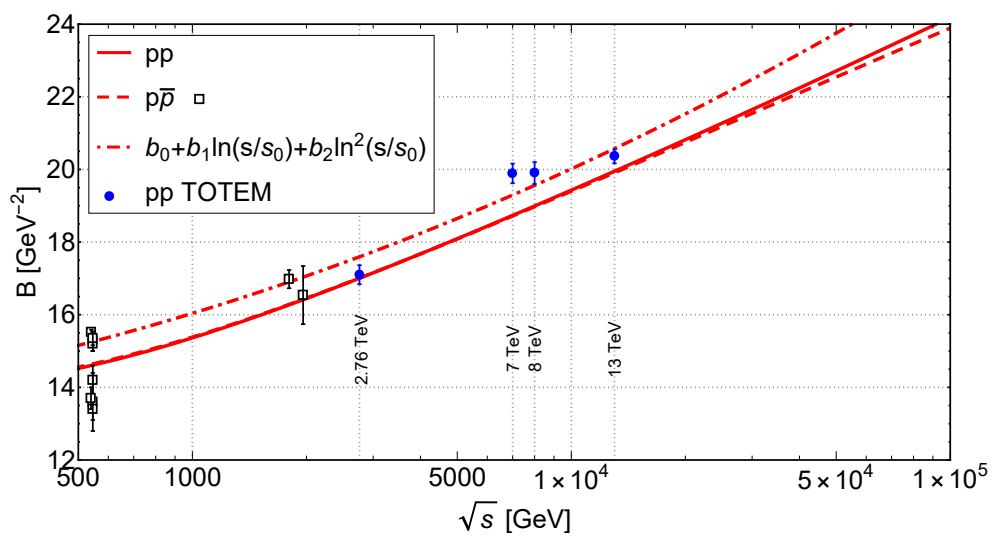

Figure 3. $p p$ and $p \bar{p}$ elastic slope $B(s)$ a) calculated from the fitted model Eqs. (1)-(6) using Eq. 8 and b) fitted with the parametrization Eq. 9 .

To see better the effect of the odderon, the deviation of $B(s)$ from its "canonical", logarithmic form, we show in Fig.4 its "normalized" shape, $B(s) /\left(a \ln \left(s / s_{0}\right)\right)$ setting $a=1 \mathrm{GeV}^{-2}$ and $s_{0}=1 \mathrm{GeV}^{2}$. A similar approach was useful in studies $[2,16]$ of the fine structure (in $t$ ) of the diffraction cone.

In Fig.4 $p p$ curve stars to increase from $\sqrt{s} \approx 50 \mathrm{TeV}$ which indicates that the slope stars to rise as $\ln ^{2} s$. Only the dipole pomeron without the odderon (dotted curve in Fig.4) at the "Born" level, fitting data on elastic, inelastic and total cross section, does not reproduce the irregular behavior of the forward slope observed at the LHC. Remarkably, the inclusion of the odderon produces a $\ln ^{2} s$ behavior of the elastic slope $B(s)$ beyond the LHC energy region.

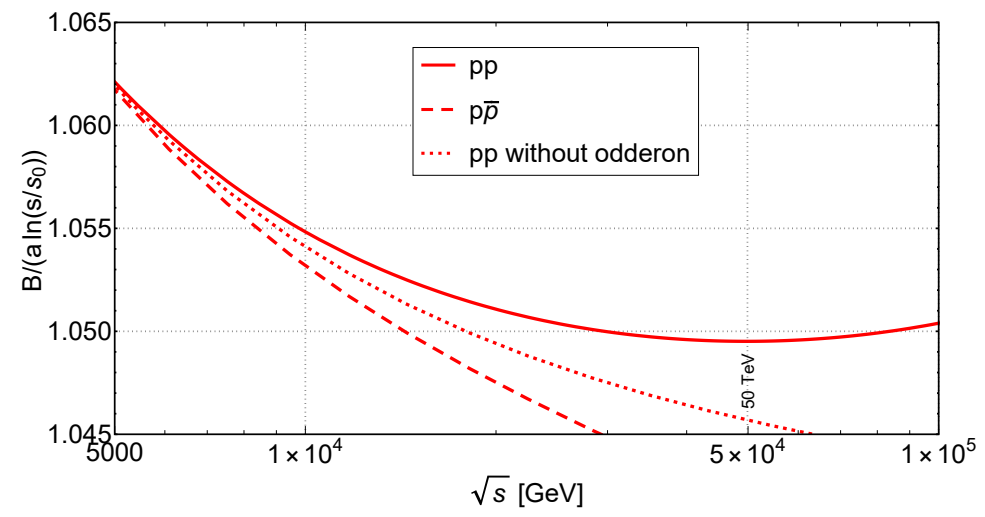

Figure 4. The ratio $B(s) /\left(a \ln \left(s / s_{0}\right)\right)$ calculated from the fitted model Eqs. (1-6) using Eq. 8 .

\subsection{The "break"}

The deviation from the linear exponential behavior was confirmed by recent measurements by the TOTEM Collaboration at the CERN LHC, first at $8 \mathrm{TeV}$ (with a significance greater than $7 \sigma$ ) [2] and subsequently at $13 \mathrm{TeV}$ [3]. 
At the ISR the "break" was illustrated by plotting the local slope [17]

$$
B(t)=\frac{d}{d t} \ln (d \sigma / d t)
$$

for several $t$-bins at fixed values of $s$. At the LHC, the effect is of the same order of magnitude and is located near the same value of $-t \approx 0.1 \mathrm{GeV}^{2}$. Different from the ISR the TOTEM quantifies the deviation from the exponential by normalizing the measured cross section to a linear exponential form $[2,3]$.

The normalized form as used by TOTEM:

$$
R(t)=\frac{d \sigma / d t}{d \sigma / d t_{r e f}}-1,
$$

where $d \sigma / d t_{r e f}=A e^{B t}$, with $A$ and $B$ are constants determined from a fit to the experimental data.

The observed "break" can be identified [18] with a two-pion exchange effect. As shown by Barut and Zwanziger [19], $t$-channel unitarity constrains the Regge trajectories near the threshold, $t \rightarrow t_{0}$ by

$$
\operatorname{Im} \alpha(t) \sim\left(t-t_{0}\right)^{\mathfrak{R} e \alpha\left(t_{0}\right)+1 / 2},
$$

where $t_{0}$ is the lightest threshold, $4 m_{\pi}^{2}$ in the case of the vacuum quantum numbers (pomeron or $f$ meson). Since the asymptotic behavior of the trajectories is constrained by dual models with Mandelstam analyticity by square-root (modulus $\ln t$ ): $\left|\frac{\alpha(t)}{\sqrt{t} \ln t}\right|_{t \rightarrow \infty} \leq$ const, (see Ref. [18] and references therein), for practical reasons it is convenient to approximate, for the region of $t$ in question, the trajectory as a sum of square roots. Higher thresholds, indispensable in the trajectory, may be approximated by their power expansion, i.e. by a linear term, matching the threshold behavior with the asymptotic.

By using a simple Regge-pole model with two leading (pomeron and odderon) and two secondary reggeons, $f$ and $\omega$ exchanges we have mapped the "break" fitted at the ISR onto the LHC TOTEM 8 and $13 \mathrm{TeV}$ data. The model, the detailed results of fits and the parameters are presented in Ref [16]. In normalized form, Fig. 5 and Fig. 6 show our description to the "break" measured at 8 and $13 \mathrm{TeV}$. These results re-confirm the earlier finding that the "break" can be attributed the presence of two-pion branch cuts in the Regge parametrization.

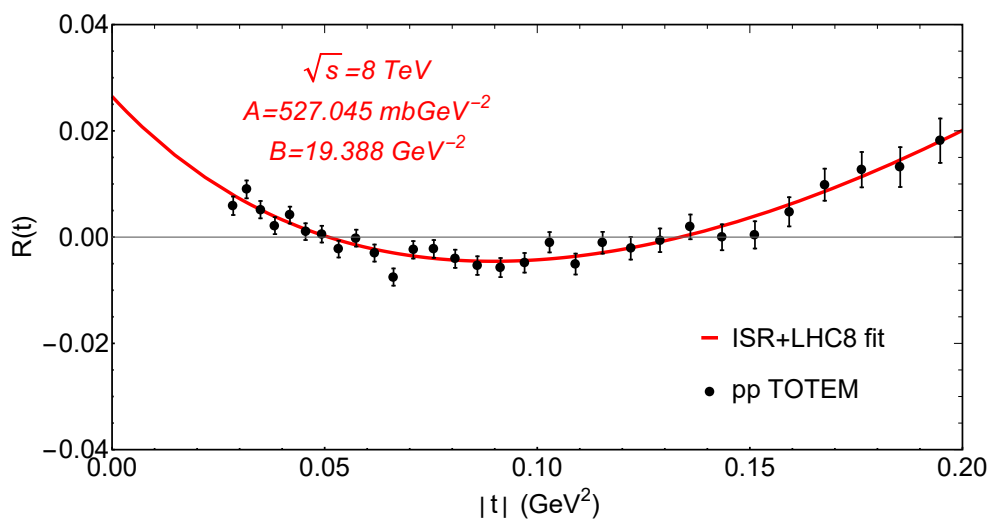

Figure 5. Normalized differential cross section $R(t)$ calculated from low- $|t| 8 \mathrm{TeV}$ TOTEM data [2] using Eq. (11). This figure is from Ref. [16]. 


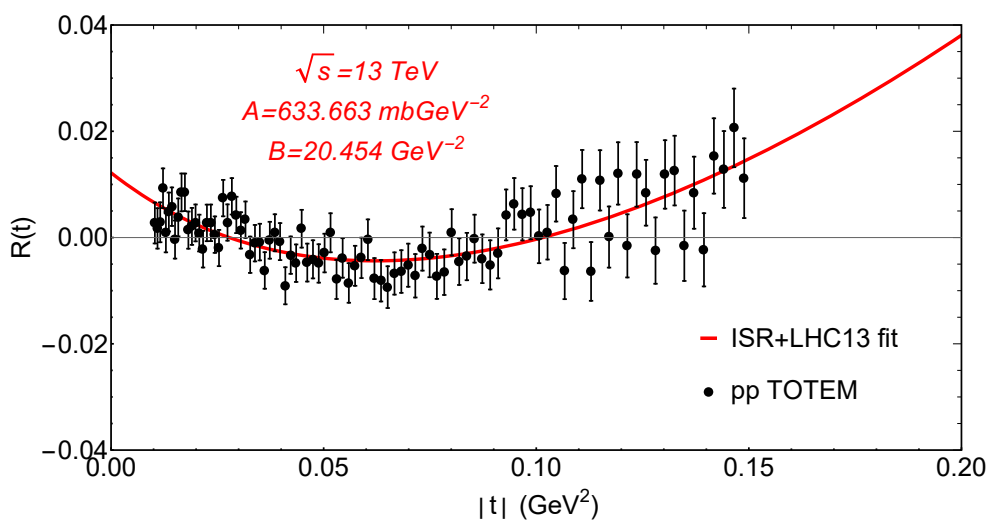

Figure 6. Normalized differential cross section $R(t)$ calculated from low- $|t| 13 \mathrm{TeV}$ TOTEM data [3] using Eq. (11). This figure is from Ref. [16].

In Ref [16] two further aspects of the phenomenon were investigated, namely: 1) to what extent is the "break" observed recently at the LHC a "recurrence" of that seen at the ISR (universality)? 2) what is the relative weight of the Regge residue (vertex) compared to the trajectory (propagator) in producing the "break"? We showed that the deviation from a linear exponential of the $p p$ diffraction cone as seen at the ISR and at the LHC are of similar nature: they appear nearly at the same value of $t \approx-0.1 \mathrm{GeV}^{2}$, have the same shape of comparable size, and may be fitted by similar $t$-dependent function. Furthermore we find that the Regge residue and the pomeron trajectory have nearly the same weight and importance.

\subsection{The diffraction minimum and maximum (dip-bump)}

The most sensitive (crucial) test for any model of elastic scattering is the well-known dipbump structure in the differential cross section. It was measured in a wide range of energies and squared momenta transfers. None of the existing models was able to predict the position and dynamics of the dip for (especially when both $p p$ and $\bar{p} p$ data are included). The first LHC measurements (at $7 \mathrm{TeV}$ ) [4] clearly demonstrated their failure. Recently TOTEM made public [3] new, preliminary data on elastic differential cross section at highest LHC energy $13 \mathrm{TeV}$ extending up to $t=-3.5 \mathrm{GeV}^{2}$. The main message from these data is the second cone is smooth, structureless. This fact excludes most of the models, where the dip is created by unitarization resulting in interference between single and multiple scattering or, alternatively by eikonal) corrections, generating multiple diffraction minima and maxima.

Before going into details, let us remind that the present DP model predicts $d \sigma /\left.d t\right|_{\text {min. }} \sim$ $1 / L, d \sigma /\left.d t\right|_{\text {max. }} \sim L$ and consequently $\frac{d \sigma /\left.d t\right|_{\text {max. }}}{d \sigma / d t_{\text {min. }}} \sim L^{2}$, where $L=\ln s$, in the case of a single pomeron contribution. The addition of the odderon, due to its opposite sign destroys the dip in case of $\bar{p} p$ scattering (degrading to a "shoulder" at the dip position). In $p p$ the odderon contributes with the same sign as the pomeron and their overall result may depend on the details or the parametrization. We have performed a fit of the differential cross section in the dip region with both the pomeron and odderon.

The results of our fit using the model Eqs. (1)-(6) for $p p$ and $p \bar{p}$ differential cross section data are shown in Fig. 7. 


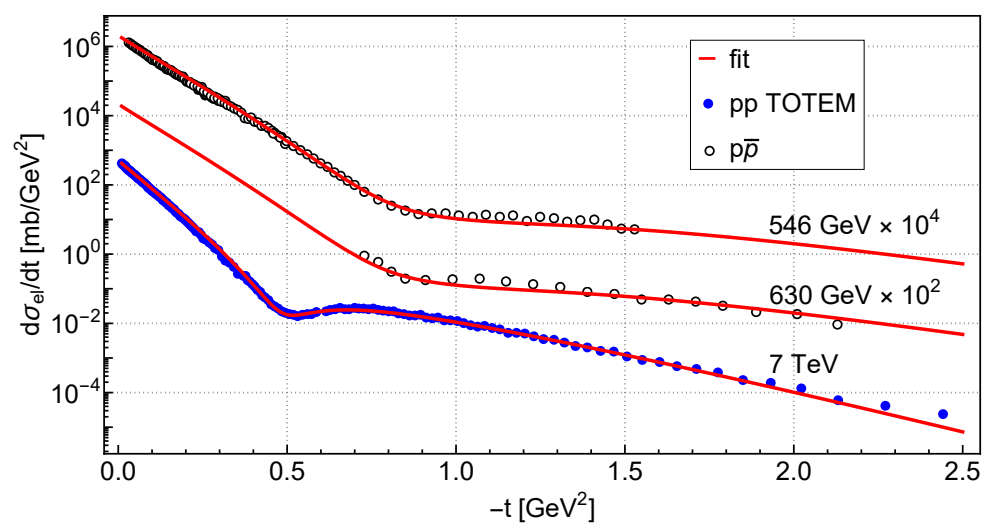

Figure 7. Results of the fit for $p p$ and $p \bar{p}$ differential cross section data $[4,10]$ using the model Eqs. (1$6)$.

Next we study the energy dependence of the minimum and maximum of $\mathrm{pp}$ diffraction cone i.e. the behavior of $\left(d \sigma_{e l} / d t\right)_{\min }(s),\left(d \sigma_{e l} / d t\right)_{\max }(s)$ and their ratio $\left(d \sigma_{e l} / d t\right)_{\max }(s) /\left(d \sigma_{e l} / d t\right)_{\min }(s)$. The mentioned quantities were calculated numerically from the fitted model and they are plotted in Fig. 8 and Fig. 9. At high energies $(\sqrt{s} \gtrsim 500 \mathrm{GeV})$ in the dip-bump region the secondary reggeons can be neglected and thus here we investigate only the contributions of the pomeron and the odderon in the evolution of the minimum and maximum.

Fig. 9 shows that in the ratio $\left(d \sigma_{e l} / d t\right)_{\max }(s) /\left(d \sigma_{e l} / d t\right)_{\min }(s)$ the pomeron and the odderon components separately monotonically increase which means that both produce monotonically deepening minimum with increasing energy. However, the ratio $\left(d \sigma_{e l} / d t\right)_{\max }(s) /\left(d \sigma_{e l} / d t\right)_{\min }(s)$ monotonically decreases in the case when both the pomeron and the odderon components are present. To summarize, our conclusion about the dynamics of the dip-bump structure at LHC energies is inconclusive. Further studies, both theoretical and experimental of this important and delicate phenomenon are necessary.

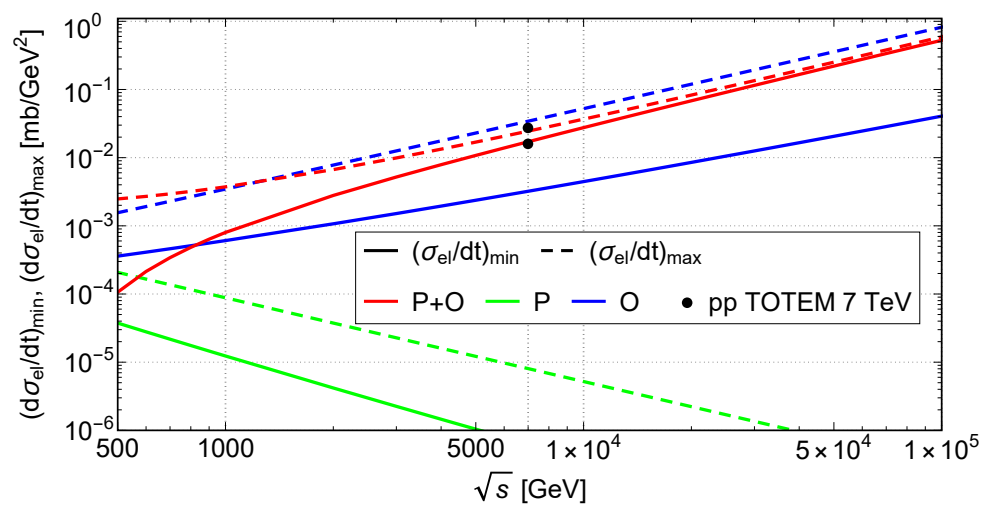

Figure 8. Energy dependence of the maximum and minimum of the $p p$ diffraction cone calculated from the fitted model. 


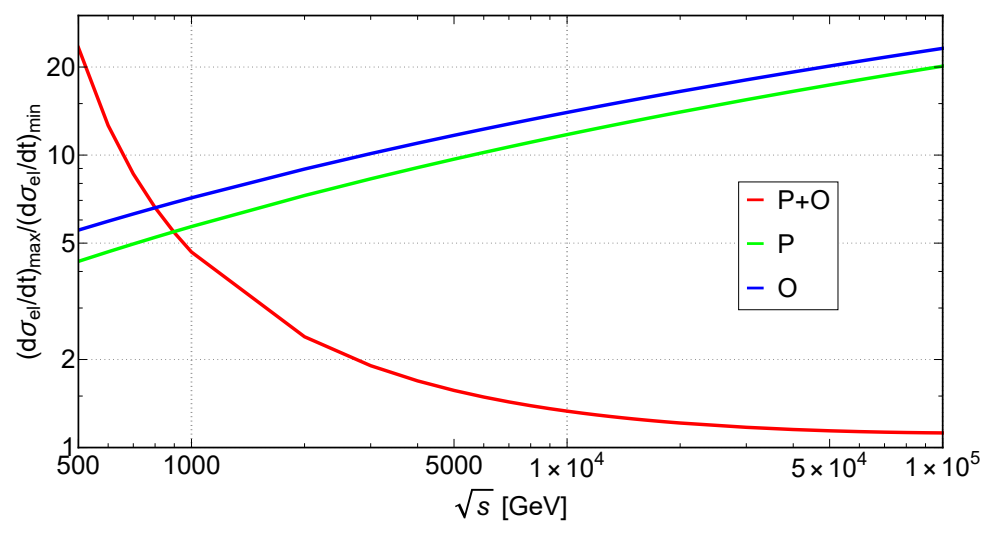

Figure 9. Energy dependence of the ratio of the maximum and minimum of the $p p$ diffraction cone calculated from the fitted model.

\section{Conclusions}

We conclude that:

1. The "break" is a universal feature of the forward cone. Regge-pole models interpolate this effect from the ISR energies up to those of the LHC. The "break" is due to the non-linear behavior of the pomeron trajectory and the mon-exponential Regge residue, both resulting from a threshold singularity in the amplitude due to $t$-channel unitarity.

2. A single diffraction minimum (and maximum) in $d \sigma / d t$ is produced by a particular interference between a single and double pomeron poles. Unitarization, for example eikonalizaiton produces multiple dips and bumps. We did no include two rightmost data points $\left(-t>2.25 \mathrm{GeV}^{2}\right)$ lying above the exponential curve; probably, they indicate the transition from "soft" to "hard" scattering, that in our model is mimicked by the transition from the linear to logarithmic regime of the Regge (here, pomeron) trejectory (see e.g. [8] and erlier references therein).

3. The observed non-monotonic rise of the slope $B(s, t)$ at the LHC is incompatible with a single pomeron pole. Even the combination of a simple and double pole (DP) cannot accommodate for the accelerated rise of $B(s)$ at the LHC. The $\log ^{2} s$ increase of the forward slope $B(s)$, reported by TOTEM, however may be reproduced by the odderon contribution to the scattering amplitude. The odderon may play and important role in the behaviour of $B(s)$ at high energies, as shown in Fig. 4.

4. The recently reported low $\rho(13)=0.1$ data point was not predicted but it was adjusted, (together with the total cross section data) in several papers published after the appearance of the experimental data, thus they should be considered as "proofs" of the odderon, whose existence was never questioned. Critical will be fits including the dip-bump region, especially for various energies and both for $p p$ and $\bar{p} p$ scattering. In any case, confirmation of this the value $\rho(13)=0.1$ by an independent experimant is welcome.

An even more important consequence for the dynamics, than any particular parametrization of the odderon, is that it may indicate (anticipate) the future slow-down (due to saturation effects) of the rise of cross sections with energy. 


\section{Acknowledgments}

The work of N. Bence and I. Szanyi was supported by the "Márton Áron Szakkollégium" program.

\section{References}

[1] TOTEM Collab. (G. Antchev et al.), First measurement of elastic, inelastic and total cross-section at $\sqrt{s}=13 \mathrm{TeV}$ by TOTEM and overview of cross section data at LHC energies, CERN-EP-2017-321.

[2] G. Antchev et al. (TOTEM Collab.), Nucl. Phys. B 899, 527 (2015), arXiv:1503.08111.

[3] The TOTEM collaboration, (2017), https://cds.cern.ch/record/2298154, to be publ. in Phys. Rev. D.

[4] G. Antchev et al. (TOTEM Collab.), Europhys. Lett. 101, 21002 (2013).

[5] G. Aad et al. (ATLAS Collab.), Nucl. Phys. B 889, 486 (2014).

[6] M. Aaboud et al. (ATLAS Collab.) Phys. Lett. B 761, 158 (2016).

[7] A.N. Wall, L.L. Jenkovszky, and B.V. Struminsky, Sov. J. Particles and Nuclei, 19, 180 (1988).

[8] L.L. Jenkovszky, A.I. Lengyel, D.I. Lontkovsky, Int'1 J. Mod. Phys. A, 26, 4755 (2011), $\operatorname{arXiv/1105.1202.~}$

[9] S. Donnachie, G. Dosch, P. Landshoff and O. Nachtmann, Pomeron physics and QCD (Cambridge University Press, 2002).

[10] http://durpdg.dur.ac.uk

[11] G. Antchev et al. (TOTEM Collab.), Europhys. Lett. 101, 21004 (2013).

[12] C. Patrignani et al. (Particle Data Group), Chin. Phys. C, 40, 100001 (2016).

[13] G. Antchev et al. (TOTEM Collab.), Phys. Rev. Lett. 111, 012001 (2013).

[14] G. Antchev et al. (TOTEM Collab.), Eur. Phys. J. C 76, 661 (2016), arXiv:1610.00603.

[15] P. Abreu et al. (Pierre Auger Collab.) Phys. Rev. Lett. 109, 062002 (2012), arXiv:1208.1520.

[16] L. Jenkovszky, I. Szanyi, C.-I Tan, Eur. Phys. J. A (2018) 54:116, arXiv:1710.10594.

[17] G. Barbiellini et. al. Phys. Letters B39 (1972) 663.

[18] G. Cohen-Tannoudji, V.V. Ilyin, Laszlo L. Jenkovszky, Nuov. Cim. 5, 957 (1972).

[19] A.O. Barut and D.E. Zwanziger, Phys. Rev. 127, 974 (1962). 